\title{
Analysis, Design, and Implementation of Intelligent Fuzzy Expert System for Marine Wealth Preservation
}

\author{
Yasser A. Nada \\ Computer Science Dept. \\ Faculty of CIT, Taif University \\ Taif, Saudi Arabia
}

\author{
Yasser H. Elawady \\ Information Technology Dept., \\ Faculty of CIT, Taif University \\ Taif, Saudi Arabia
}

\begin{abstract}
Fishing has become a major threat to marine fishes. Effective conservation requires timely identification of vulnerable fish species. However, evaluation of extinction risk using conventional methods is difficult due to limitations in data that should be gathered about the fish species and required by such methods. This paper presents a fuzzy expert system that integrates life history and ecological characteristics of marine fishes to estimate their intrinsic vulnerability. There are lots of general and special purpose expert systems that help society in a life particular sector. So, a professional one is selected and adapted for helping in marine wealth preservation. Finally, the proposed fuzzy expert system is used as a decision support tool in fishery management and marine conservation planning.
\end{abstract}

\section{General Terms}

Fuzzy Set Theory engines; Expert System

\section{Keywords}

Expert System; Forward Chaining; CLIPS; Marine Wealth Preservation; Fuzzy Set Theory engines.

\section{INTRODUCTION}

Expert systems (ES) are a branch of applied artificial intelligence involving that expertise, which is the vast body of task-specific knowledge, is transferred from a human to a computer. Expert systems can be used as a solution to overcome the scarcity of experts.

Expert systems can be applied to almost any human endeavor that requires expertise. They are a natural outgrowth of heuristics-based chess-playing programs in which the expertise of chess masters was incorporated into the programs. Computer scientists began to use the same technique in other areas of human knowledge that require expert knowledge, such as medicine, engineering, and law. In the field of medicine, expert systems using the diagnostic skills of physicians can be designed to analyze the symptoms of a patient and to diagnose medical problems.

EurOcean 2014 was convened by the Italian Presidency of the Council of the European Union, the European Marine Board, the European Commission and three esteemed Italian partner institutions: The National Research Council, National InterUniversity Consortium for Ocean Science (CoNISMa) and the National Institute of Oceanography and Experimental Geophysics (OGS). In addition to a rising tide of oceanrelated threats to human health and economics, the conference statement points to major opportunities in such areas as marine biotechnology, offshore energy, and sustainable aquaculture to create much-needed jobs after one of the worst economic crises in recent history [1].
The ocean resources in the methodological sense is not only the physical properties of the water space, but the ocean, as a reservoir, taking into account the size, geographical and climatic characteristics, including any useful properties of the marine environment the development activity of the investor is directed to, and among them the activity developments at the sea and the coasts, the economic potential of marine cargo transportations, the ocean biological resources, the oceanbottom wealth at the shelf and behind its limits, the marine energy resources, the recreational properties, etc[2].

Fishing has become a major conservation threat to marine fishes. Effective conservation of threatened species requires timely identification of vulnerable species. However, evaluation of extinction risk using conventional methods is difficult for the majority of fish species because the population data normally required by such methods are unavailable. This paper presents a fuzzy expert system that integrates life history and ecological characteristics of marine fishes to estimate their intrinsic vulnerability to fishing. We extract heuristic rules (expressed in IF-THEN clauses) from published literature describing known relationships between biological characteristics and vulnerability. Input and output variables are defined by fuzzy sets which deal explicitly with the uncertainty associated with qualitative knowledge. Conclusions from different lines of evidence are combined through fuzzy inference and defuzzification processes. Our fuzzy system provides vulnerability estimates that correlate with observed declines more closely than previous methods, and has advantages in flexibility of input data requirements, in the explicit representation of uncertainty, and in the ease of incorporating new knowledge. This fuzzy expert system can be used as a decision support tool in fishery management and marine conservation planning.

The solution for all these problems is through an expert system includes the necessary expertise in this field. It provides the tips are derived from expertise's of experts in this field, through magazines, articles of a famous personalities.

\section{LITERATURE SURVEY}

\subsection{The Value of Marine Ecosystem}

The world's oceans are not only the domain of food for human being but also the legitimate concern of marine transport, offshore extraction of oil, gas and other minerals, climate control and recreation. Marine fisheries account for $85 \%$ of the global fish catch. Maritime shipping is involved in the transport of over $80 \%$ of the world's merchandise trade. An estimated $70 \%$ of the world's fish stocks are already being exploited at or beyond sustainable limits, but fishing generally continues unabated despite extensive regulatory arrangements for their management. In a few cases, emergency measures have been taken to defend stocks of marine wildlife. In 1985, for instance, the International Whaling Commission declared 
a moratorium on commercial whaling. Time when increasing human populations are demanding more food. The decline of many world fisheries due to common access and the pressures of an increased demand for food by an ever-increasing human population have led to friction among fishing nations and various attempts to regulate fishery resources [3]

Individual fisheries have similarly been closed for prolonged periods, but the threats to fisheries remain and continue to increase.

\subsection{Division of the Marine Environment}

The marine ecosystem is the largest aquatic system on the planet. Its size and complexity make it difficult to deal with as a whole. As a result, it is convenient to divide it into more manageable arbitrary subdivisions. Open ocean can be subdivided vertically and horizontally [4].

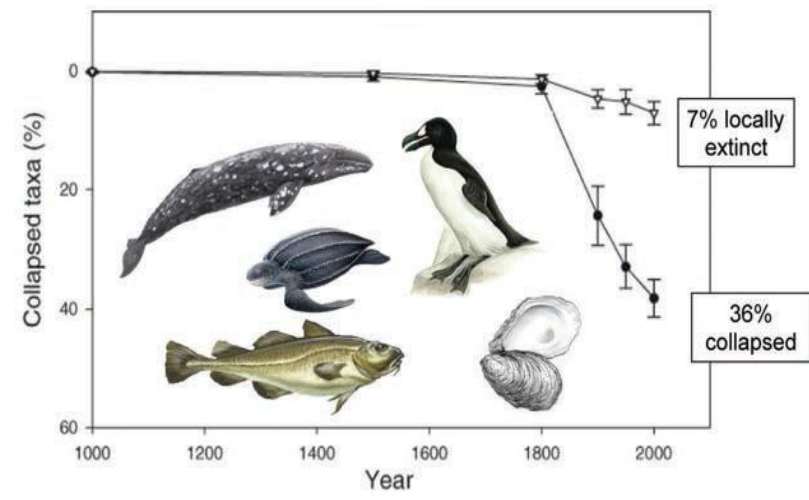

Fig 1: Long-term trend to local extinction and collapse of sea life

The Food and Agriculture Organization of the UN reports that the total tonnage of wild sea life captured by all nations has been almost flat for the past 20 years. Within the flat catch, however, specific populations have fallen and been replaced, part of a longer-term pattern of new groups becoming commercial while others collapse or even become locally extinct. These include not only fish such as cod but whales, turtles, seabirds, and oysters (Fig 1).

In fact, while humans have been getting larger, fish have been getting smaller for more than a century Long-term trend to local extinction and collapse of sea life. Source least a reasonable chance of catching a fish, a trophy, or seeing diverse life when they snorkel or scuba. Many regions have suffered terrible losses of sea life, owing to overfishing, coastal pollution, and other abuses and failed maintenance [5].

Harnessing Our Ocean Wealth is an Integrated Marine Plan (IMP), setting out a roadmap for the Government's vision, high-level goals and integrated actions across policy, governance and business to enable our marine potential to be realized. Our marine resources also provide essential noncommercial benefits such as amenity, biodiversity and our mild climate [6].

An expert system is a computer program that uses expert knowledge to attain high levels of performance in a problem area. It can be used to supplement the availability of human experts and to increase the availability of expert advice. An expert system enables anyone with some experience to outline the process used to solve a problem. Once this has been done, the outline can be used to generate a series of questions that lead a non-expert to solve environmental problems.
Expert systems have been used to solve a wide range of problems in domains such as medicine, mathematics, engineering, geology, computer science, business, law, defense and education. Building an expert system therefore first involves extracting the relevant knowledge from the human expert. Such knowledge is often heuristic in nature, based on rules rather than absolute certainties [7].

Extracting knowledge from the expert in a way that can be used by a computer is generally a difficult task. Experts generally find it very difficult to express exactly what knowledge and rules they use to solve a problem. i.e., knowledge acquisition for expert systems is a big area of research, with a wide variety of techniques developed. In this paper, the technique for extracting production rules then using these rules as knowledge base of expert systems is based on IF-THEN rules [8].

\section{COMPONENTS OF AN EXPERT SYSTEM}

Expert System (ES), also called a Knowledge Based System (KBS), is computer application programs that take the knowledge of one or more human experts in a field and computerize it so that it is readily available for use. It can also be integrated with textual database which can be used for explanation purposes of basic terms and operations to confirm and to reach conclusion in some situations [9].

One of the most powerful attributes of expert systems is the ability to explain reasoning. Since the system remembers its logical chain of reasoning, a user may ask for an explanation of a recommendation and the system will display the factors it considered in providing a particular recommendation. This attribute enhances user confidence in the recommendation and acceptance of the expert system [10].

All expert systems are composed of several basic components: a user interface, a database, a knowledge base, and an inference mechanism. Moreover, expert system development usually proceeds through several phases including problem selection, knowledge acquisition, knowledge representation, programming, testing and evaluation. Expert systems have a number of major system components and interface with individuals in various roles. These are illustrated in Fig (2). The major components are [11]:

- Knowledge base - a declarative representation of the expertise, often in IF THEN rules;

- Working storage - the data which is specific to a problem being solved;

- Explanation facility - the explanation facility tells the user how the conclusion was reached, and what facts and rules were used. Thus a professional user can evaluate the approach for its logic and correctness.

- Domain expert - the individual or individuals who currently are experts solving the problems that the system is indented to solve.

- Knowledge engineer - the individual who encodes the expert's knowledge in a declarative form that can be used by the expert system.

- User- the individual who will be consulting with the system to get advice which would have been provided by the expert. 
- System engineer - the individual, who builds the user interface, designs the declarative format of the knowledge base and implements the interface engine.

- Inference engine - the code at the core of the system which derives recommendations from the knowledge base and problem-specific data in working storage;

- User interface - the code that controls the dialog between the user and the system.

Fig 2 explains the Architecture of the Expert System.

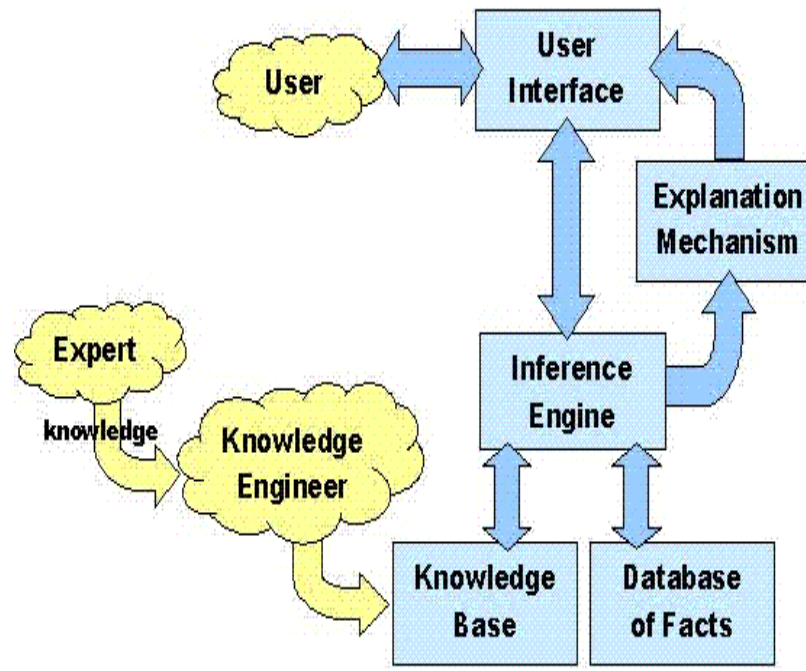

Fig 2 : The Expert System Component

\section{CHARACTERISTICS OF AN EXPERT SYSTEM}

The following are characteristics of an expert system:

- Can explain their reasoning or suggested decisions. Users can understand how and why a conclusion was reached.

- Can display "intelligent" behavior. It proposes new ideas from the data.

- Can draw conclusions from complex relationships. This is the ability to evaluate relationships to solve problems.

- Can provide portable knowledge. When a human expert leaves an organization, the expert's knowledge and methods for problem solving are generally no longer available. An expert system can capture that expert's knowledge and approach to problem solving.

- Can deal with uncertainty. An expert system uses probability and heuristics to cope with incomplete data.

\section{KNOWLEDGE REPRESENTATION}

The key problem is to find a KR (and a supporting reasoning system) that can make the inferences your application needs in time, that is, within the resource constraints appropriate to the problem at hand. This tension between the kinds of inferences and application "needs" and what counts as "in time" along with the cost to generate the representation itself makes knowledge representation engineering interesting.
There are representation techniques such as frames, rules, tagging, and semantic networks which have originated from theories of human information processing. Since knowledge is used to achieve intelligent behavior, the fundamental goal of knowledge representation is to represent knowledge in a manner as to facilitate inference (i.e. drawing conclusions) from knowledge $[12,13]$.

Knowledge bases can be represented by production rules. These rules consist of a condition or premise followed by an action or conclusion (IF condition...THEN action).

\section{FUZZY LOGIC}

The classical set theory is built on the fundamental concept of "set" of which an individual is either a member or not a member. A sharp, crisp, and unambiguous distinction exists between a member and a nonmember for any well-defined "set" of entities in this theory, and there is a very precise and clear boundary to indicate if an entity belongs to the set. In other words, when one asks the question "Is this entity a member of that set?" The answer is either "yes" or "no." This is true for both the deterministic and the stochastic cases. In probability and statistics, one may ask a question like "What is the probability of this entity being a member of that set?" In this case, although an answer could be like "The probability for this entity to be a member of that set is $90 \%$," the final outcome (i.e., conclusion) is still either "it is" or "it is not" a member of the set. The chance for one to make a correct prediction as "it is a member of the set" is $90 \%$, which does not mean that it has $90 \%$ membership in the set and in the meantime it possesses $10 \%$ non-membership. Namely, in the classical set theory, it is not allowed that an element is in a set and not in the set at the same time. Thus, many real-world application problems cannot be described and handled by the classical set theory, including all those involving elements with only partial membership of a set. On the contrary, fuzzy set theory accepts partial memberships, and, therefore, in a sense generalizes the classical set theory to some extent. To see More details in Fuzzy Set Theory is found in [14].

\section{INFERENCE ENGINE}

The Inference Engine forms the heart of the expert system; the knowledge base serves as the brain of the expert system. The inference engine chums through countless potential paths and possibilities based on some combinations of rules, cases, models or theories [15].

Two methods of inference often are used, forward and backward chaining. Forward chaining is a top down method which takes facts from satisfied conditions in rules which lead to actions being executed. Backward chaining is the reverse. It is a bottom up procedure which starts with goals (or actions) and queries the user about information which may satisfy the conditions contained in the rules [16].

\subsection{Expert System Shell}

Expert System Shell is a software package containing a generic inference engine, a user interface, and a collection of other tools that enable users to develop and use expert systems.

The development of expert system is implemented in CLIPS programming environment (C) Language Integrated Production System) [17-19]. This programming tool is designed to facilitate the development of software to model human knowledge or expertise. CLIPS program is used by reason of the flexibility, the expandability and the low cost. CLIPS keep in memory a fact list, a rule list, and an agenda 
with activations of rules. Facts in CLIPS are simple expressions consisting of fields in parentheses. Groups of facts in CLIPS, usually follow a fact-template, so that to be easy to organize them and thus design simple rules that apply to them.

The entire control and operation of the system are done by the inference engine; that is developed using $\mathrm{C \#}$; The main roles of the inference engine are summarized as: It applies the expert domain knowledge to what is known about the present situation to determine new information about the domain. The inference engine is the mechanism that connects the user inputs in the form of answers to the questions to the rules of knowledge base and further continues the session to come to conclusions. This process leads to the solution of the problem.

We will elicit knowledge from marine conservation experts who has experience in the marine wealth preservation. We must elicit this knowledge in accurate and complete manner.

\subsection{Forward Chaining}

Forward Chaining is a tracking process that begins with displaying a collection of data or evidence convincing to the final conclusion [20]. Forward chaining also called a search driven data. So starting from the input information (if) first and then to the conclusion (derived information) (then) that can be modeled as follows:

\section{IF (input information) \\ THEN (conclusion)}

So that the Forward Chaining logic can be started from the data to the destination, from the findings to the explanation, and/or from the observations to the diagnosis. Example for Forward Chaining tracking process in the form of production rules:

\section{RULE}

Question " does your animal have a flat body?"

If phylum is elsewhere and body in segments is yes

Then class is segments

If phylum is elsewhere and body in segments is no

Then class is unified

Question " does your animal eat red meat?"

If class is water and boney is yes

Then type animal is fish

If class is water and boney is no

Then type animal is shark/ray

\section{RESULTS AND TESTING THE SYSTEM}

The system was evaluated with different users, including developers, and staff. The system has validated by experts in the domain of marine wealth. Tests of the system were carried out by the developers to make sure the system would work correctly. Fig's $(3,4,5,6$ and 7$)$ shows the snapshots of the developed system.

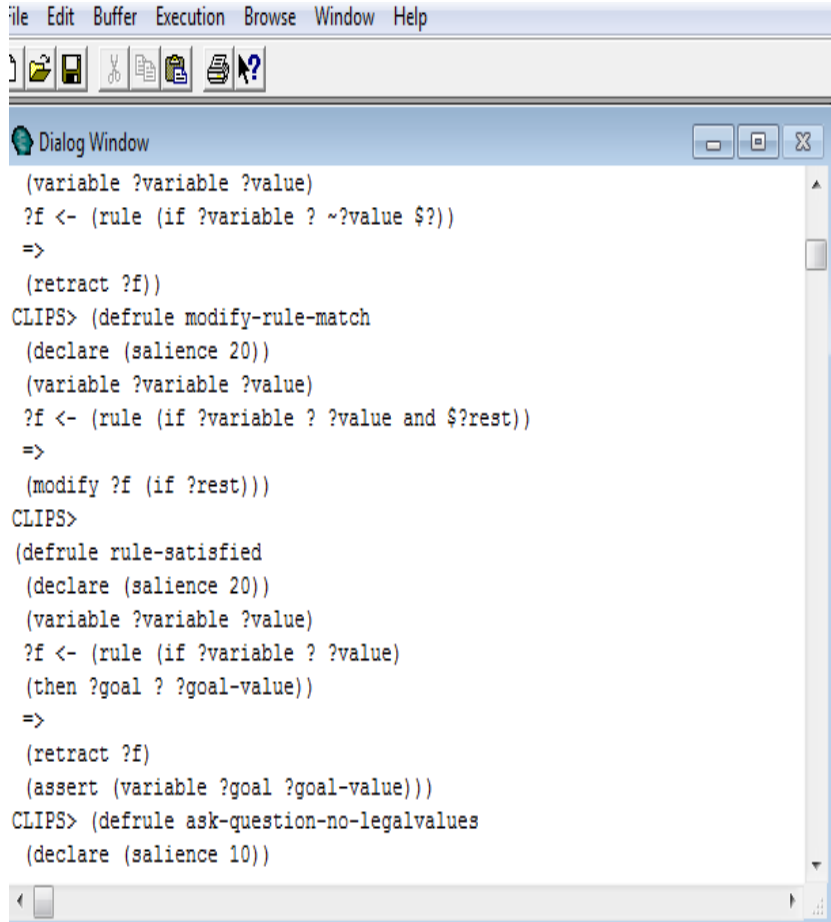

Fig 3 : Domain Selection Screen

ile Edit Buffer Execution Browse Window Help

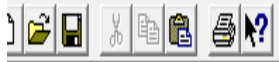

Oialog Window

(rule (if superphylum is jellyback and

live.prime.in.soil is no)

(then phylum is elsewhere))

(question live.prime.in.soil is "Does your animal live primarily in soil (rule (if phylum is warm and

has.breasts is yes)

(then class is breasts))

(rule (if phylum is warm and

has.breasts is no)

(then type.animal is bird/penguin))

(question has.breasts is "Normally, does the female of your animal nurse (rule (if phylum is cold and

always,in.water is yes)

(then class is water))

(rule (if phylum is cold and

always, in.water is no)

(then class is dry))

(question always, in.water is "Is your animal always in water?") (rule (if phylum is soil and

flat.bodied is yes)

(then type.animal is flatworm))

Fig 4 : Run the System 


"lle talt butter txecution browse window Help
Doialog Window
boney is yes)
(then type,animal is fish))
(rule (if class is water and
boney is no)
(then type,animal is shark/ray))
(question boney is "Does your animal have a boney skeleton?")
(rule (if class is dry and
scally is yes)
(then order is scales))
(rule (if class is dry and
scally is no)
(then order is soft))
(question scally is "Is your animal covered with scaled skin?")
(rule (if class is segments and
shell is yes)
(then order is shell))
(rule (if class is segments and
shell is no)
(then type.animal is centipede/millipede/insect))
(question shell is "Does your animal have a shell?")
(rule (if class is unified and
1 D

Fig 5 : Run the System

ille Edit Buffer Execution Browse Window Help

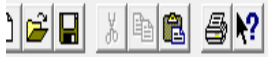

O Dialog Window

(then type.animal is flatworm)

(rule (if phylum is soil and

flat.bodied is no)

(then type,animal is worm/leech))

(question flat.bodied is "Does your animal have a flat body?")

(rule (if phylum is elsewhere and

body. in. segments is yes)

(then class is segments))

(rule (if phylum is elsewhere and

body. in. segments is no)

(then class is unified))

(question body.in. segments is "Is the animals body in segments?")

(rule (if class is breasts and

can. eat.meat is yes)

(then order is meat))

(rule (if class is breasts and

can. eat.meat is no)

(then order is vegy))

(question can.eat.meat is "Does your animal eat red meat?")

(rule (if class is water and

boney is yes)

Oile Edit Buffer Execution Browse Window Help
(answer is "I think your animal is a " type.animal))
CLIPS> (reset)
CLIPS> (run)
Does your animal have a backbone? (yes no) yes
Is the animal warm blooded? (yes no) no
Is your animal always in water? (yes no) no
Is your animal covered with scaled skin? (yes no) yes
Does the animal have a rounded shell? (yes no) yes
I think your animal is a turtle
CLIPS> (reset)|
CLIPS> (run)|
Does your animal have a backbone? (yes no) no
Does your animal live primarily in soil? (yes no) yes
Does your animal have a flat body? (yes no) yes
I think your animal is a flatworm
CLIPS> |
1 $\square$ (

Fig 7: Final Result

\section{CONCLUSIONS}

In this paper, a fuzzy expert system, which considered as a decision support tool in fishery management and marine conservation planning, is proposed. In the proposed fuzzy expert system, the fuzzy set theory is used to implement the vague knowledge representation with fuzzy logic to perform the inferences. Moreover, the proposed fuzzy expert system, illustrate examples to demonstrate the need for, and the implementation of, fuzzy set theory in a marine wealth system. This expert system is constructed by using Forward Chaining method in addition to acquiring expert's knowledge and CLIPS, which in turn makes the proposed system intelligent.

\section{REFERENCES}

[1] Terry Collins, "Human health, wealth require expanded marine science", EurOcean 2014 conference, In Rome Italy, 2014.

[2] Michael V. Voyloshnikov, "Evaluation of Commercial Vessels, Marine Company's Assets and Ocean Resources", Reviewers: Eugeny A.Gnezdilov, Ph.D., Honorable economist of the Russian Federation Valeria V.Krivets (Goncharova), Ph.D., Certificated appraiser of RSA.

[3] AN. Subramanian, "Introduction : Marine Environment", Centre for Marine Environmental Studies, Ehime University, Japan.

[4] Hedgepeth, J., "Classification of marine environments and concepts of marine ecology", In: Hedgepeth, J.E. (Ed.), The treatise on marine ecology and paeoecology. Vol. 1, Ecology. Memoir No. 67 of the Geological society of America, New York,1957.

[5] Jesse H. Ausubel., "Wealth from the Oceans Use, Stewardship, and Security", Keynote Address, 8th Future of the Ocean Symposium Urban Coast Institute, Monmouth University, 26 October 2012.

[6] Enda Kenny, T.D., Simon Coveney, T.D., "Harnessing Our Ocean Wealth", An Integrated Marine Plan for Ireland Roadmap, New Ways, New Approaches, New Thinking, 2012. 
[7] Sarhan, A. M., W. F. Abd El-Wahed, T. E. Danf, and M. M. Tokhy; " Developing a Rule Extraction Methodology for Expert System Based Artificial Neural Network Ensembles" Fourth International Conference on Informatics and Systems INFOS2006, 25- 27 March 2006.

[8] Moawad, I. F., S. Gamalel-Din, M. Zaki, and A. Rafea, “ Converting Traditional Diagnostic Expert System Into Negotiating Agent " Fourth International Conference on Informatics and Systems INFOS2006, 25- 27 March 2006.

[9] Khumukcham R., Shikhar Kr. S, "JESS Based Expert System Architecture for Diagnosis of Rice Plant Diseases: Design and Prototype Development", 2013 4th International Conference on Intelligent Systems, Modeling and Simulation, P 674-676, 2013.

[10] Tanimoto, S. L. , " The Elements of Artificial Intelligence ", Computer Science Press. , 1990.

[11] Schnupp, P.H.; "Building Expert System in Prolog ", Amiz, 2000.

[12] George F. Luger and William, A. Stubblefield; " Artificial Intelligence and the Design of Expert Systems", The Benjamin/ Cummings publishing Co., Inc., 1989.

[13] Keith D., "The Essence of Expert Systems", Prentice Hall, 2000.

[14] C. Guanrong, and P. Trung, "Introduction to Fuzzy Sets, Fuzzy Logic, and Fuzzy Control Systems", ISBN 08493-1658-8, 2001 by CRC Press LLC.

[15] Byrd, T., Cossick, K. \&Zmud, R.,"A Synthesis of Research onRequirements Analysis and Knowledge Acquisition Techniques", MISQuarterly, 16(1), 117-138, 1992.

[16] Awad, E. \& Lindgren Jr, J., " Skills and Personality Attributes ofthe Knowledge Engineer: an Empirical Study", International Associationof Knowledge Engineers '92 Proceedings, 1992.
[17] Qi Ming Cui, Sheng Wei Liu, Rong Xing Liu, and ZhiRai Wang, "Application Research of the Production System type Expert System Shell Pro/3 under Smart Grid", IEEE PES ISGT ASIA 2012.

[18] Giarranto J. C., "CLIPS User's Guide", Version 6.22, 1998.

[19] Da-peng Tan, Shi-ming JiShu-ting Chen, "Continuous Casting Slag Detection Expert System Based on CLIPS", 2010 Ninth International Symposium on Distributed Computing and Applications to Business, Engineering and Science, IEEE, 2010.

[20] Giarratano, Joseph. Riley, Gary., "Expert Systems Principles and Programming", International Thompson Publishing. 3rd Edition, 1994.

\section{AUTHOR PROFILE}

DR. Yasser Ahmed Nada Was born in Ismailia, Egypt, in 1968. He received the BSc degree in pure Mathematics and Computer Sciences in 1989 and MSc degree for his work in computer science in 2003, all from the Faculty of Science, Suez Canal University, Egypt. In 2007, he received his Ph.D. in Computer Science from the Faculty of Science, Suez Canal University, Egypt. From September 2007 until now, he worked as Assistant Professor of computer science. Chair, Department of computer science, Faculty of Computers and Information Technology, Taif University, KSA. His research interests include Expert Systems, Artificial Intelligence, Object Oriented Programming, Computer Vision, and Genetic.

DR. Yasser H. Elawady is an assistant professor in the Information Technology Dept., Faculty of Computers and Information technology, Taif University, Taif, KSA. He received his M.Sc. from the Computer Engineering Dept., Faculty of Engineering, Mansoura University, Mansoura, Egypt, in 2003. He received his Ph.D. from the Computer Science Dept., Faculty of computers and Information, Mansoura University, Mansoura, Egypt, in 2011. His subject of interest includes Remote Access, FPGA programming, Hardware Design, Computer Architecture and Organization, WSN, Networking, and Computer Vision. 